\title{
Insuficiência cardíaca: estratégias usadas por idosos na busca por qualidade de vida
}

\author{
Heart failure: strategies used by elders in search for quality of life \\ Insuficiencia cardiaca: estrategias usadas por ancianos en la búsqueda por cualidad de vida
}

\section{Analyane Conceição Silva dos Santos', Fátima Helena do Espírito Santo", Luana Pestana', Donizete Vago Daher"', Rosimere Santana'II}
' Universidade Federal Fluminense, Escola de Enfermagem Aurora de Afonso Costa, Programa de Pós Graduação em Enfermagem, Núcleo de Estudos e Pesquisas em Enfermagem Gerontológica. (Mestranda) Niterói-RJ, Brasil.
"Universidade Federal Fluminense, Escola de Enfermagem Aurora de Afonso Costa, Curso de Pós Graduação em
Enfermagem Gerontológica, Núcleo de Estudos e Pesquisas em Enfermagem Gerontológica. Niterói-RJ, Brasil.
I'I Universidade Federal Fluminense, Escola de Enfermagem Aurora de Afonso Costa,
Núcleo de Estudos e Pesquisas em Enfermagem Gerontológica. Niterói-RJ, Brasil.

Submissão: 17-04-2010 Aprovação: 28-12-2011

\section{RESUMO}

Estudo descritivo com abordagem qualitativa cujos objetivos foram identificar as repercussões da insuficiência cardíaca na qualidade de vida dos idosos; descrever as estratégias utilizadas no enfrentamento destas repercussões e conhecer as percepções acerca dos cuidados de enfermagem. Foram realizadas entrevistas semiestruturadas com dez idosos com insuficiência cardíaca em um hospital militar do Rio de Janeiro-RJ. Identificou-se que a insuficiência cardíaca gera repercussões emocionais, físicas e financeiras na vida dos idosos, ocasionando impacto na qualidade de vida. Dentre as estratégias criadas pelos idosos, verificouse o enfrentamento paliativo e o enfrentamento emocional. Os cuidados de enfermagem evidenciados foram orientação, carinho, zelo e dedicação. Cuidar do idoso com insuficiência cardíaca implica em avaliar as repercussões na capacidade funcional e autonomia dos anciãos, exigindo habilidades e competências do enfermeiro para identificar suas especificidades na perspectiva de um cuidado integral que contribua para a qualidade de vida dos mesmos.

Descritores: Idoso; Insuficiência cardíaca; Qualidade de vida; Enfermagem geriátrica.

\begin{abstract}
This is a descriptive study, with qualitative approach, aiming to identify the repercussions of heart failure on elders' quality of life; to describe the strategies used in facing these repercussions and, understand the perceptions of nursing care. Semistructured interviews were carried out with ten elders holding heart insufficiency at a military hospital in Rio de Janeiro-RJ. It was identified that heart failure generates emotional, physical and financial repercussions in the elders' lives, provoking impact on their quality of life. Among the strategies created by the elders, it was noticed the palliative coping and the emotional conflict. The nursing cares noticed were orientation, affection, goodwill and dedication. To take care of the elders holding heart failure implies in evaluating repercussions in their functional capacity and autonomy, requiring capacities and competencies of the nurses to identify their specificities in the perspective of a whole care that contributes to the elders' quality of life.
\end{abstract}

Key words: Elders; Heart failure; Life quality; Geriatric nursing.

\section{RESUMEN}

Estudio descriptivo con abordaje cualitativa cuyos objetivos fueron identificar las repercusiones provocadas por la insuficiencia cardiaca en la cualidad de vida de los ancianos, describir las estrategias usadas en lo enfrentamiento de esas repercusiones y comprender las percepciones de los cuidados de enfermería. Fueron realizadas entrevistas semiestructuradas con diez ancianos con insuficiencia cardiaca en un hospital militar en Río de Janeiro. Fue identificado que la insuficiencia cardiaca genera repercusiones emocionales, físicas y financieras en la vida de los ancianos, ocasionando impacto en la cualidad de vida. Entre las estrategias creadas por los ancianos fue verificado el enfrentamiento paliativo e el enfrentamiento emocional. Los cuidados de los enfermeros evidenciados fueron orientación, cariño, atención y dedicación. Cuidar del anciano con insuficiencia cardiaca implica en avaluar las repercusiones en la capacidad funcional y autonomía de los ancianos, exigiendo habilidades y competencias del enfermero para identificar sus especificidades en la perspectiva de un cuidado integral que contribuya para la cualidad de vida de los mismos. Palabras clave: Anciano; Insuficiencia cardiaca; Cualidad de vida; Enfermeros en geriatria geriátrica. 


\section{CONSIDERAÇÕES INICIAIS}

Com o envelhecimento progressivo da população mundial, principalmente em países em desenvolvimento, como o Brasil, aumenta a incidência de doenças cardiovasculares, dentre as quais se destaca a insuficiência cardíaca (IC) que traz repercussões para a qualidade de vida dos idosos ${ }^{(1)}$. Em geral, as pessoas com IC sofrem modificações em seu padrão de vida devido à incapacidade crescente para executar suas atividades cotidianas, decorrentes dos sinais e sintomas da doença, tais como dor, desconforto precordial, dispnéia, ortopnéia, taquicardia, síncope, fadiga e edema ${ }^{(2)}$.

Os sinais e sintomas descritos implicam em limitações e dificuldades para os indivíduos que convivem com as alterações e as restrições impostas pela doença no cotidiano, o que requer do enfermeiro preparo para realizar um cuidado que atenda não apenas as necessidades biológicas dessas pessoas, mas também as suas necessidades psicossociais ajudando-os a superar limitações e a criar estratégias de enfrentamento da doença ${ }^{(1)}$, reduzindo complicações, internações e readmissões hospitalares que além de sérias repercussões para a vida do indivíduo, oneram o sistema de saúde.

Há no Brasil cerca de dois milhões de pessoas com IC, sendo diagnosticados 240 mil casos por ano. A IC foi a causa mais frequente de internação hospitalar entre idosos no ano de 2009, atingindo mulheres e homens em proporções semeIhantes $^{(3)}$. Este dado reforça a magnitude do problema que esta patologia cardíaca representa para o sistema de saúde, seus portadores e toda a sociedade, sendo desta forma, tema de estudo de muitas pesquisas.

A IC é considerada uma síndrome que é, ao mesmo tempo, complexa e comum, com aspectos clínicos multifatoriais que geram repercussões sociais devido a sua morbidade e mortalidade $^{(4)}$, sendo definida como a incapacidade do coração em bombear sangue suficiente para satisfazer as necessidades de oxigênio e nutrientes do organismo. As pessoas com IC sofrem de vários sintomas, muitos dos quais não são específicos e, frequentemente, resultam em redução da capacidade funcional e piora da qualidade de vida, ambos relacionados com a dispneia e fadiga durante as atividades diárias ${ }^{(5)}$.

O conceito de qualidade de vida (QV) é algo particular, varia de acordo com as experiências vivenciadas ao longo da vida podendo ser definido de maneira diferente por uma mesma pessoa dependendo da fase da vida em que a mesma esteja. No caso da velhice, a qualidade de vida está intimamente relacionada à manutenção da autonomia, sendo esta percebida no desempenho das atividades de vida diária ${ }^{(17)}$.

O conceito de QV sofre alterações com o surgimento de uma doença, principalmente se ela for crônica, como é a IC, visto que o indivíduo terá que conviver com esta por toda sua vida. Isso significa que, sendo um processo crônico, implica na necessidade do individuo de fazer mudanças no estilo de vida e de continuidade do tratamento o que envolve acompanhamento das diversas fases da doença, caracterizadas por manifestações clinicas em períodos de remissão e exacerbação em graus variados de incapacidade e morte(6).

As doenças cardíacas, e, entre estas, a IC, despontam como coadjuvantes em termos de importância na atenção à saúde do idoso, pois sua incidência tende a aumentar com o avançar da idade. Uma vez instalada, a IC, devido às limitações decorrentes dos sintomas físicos e psicológicos associados, apresenta forte impacto na vida da pessoa. Entre os sintomas psicológicos da IC destacam-se o medo, a insegurança e a tristeza que pioram com o agravamento progressivo da doença( ${ }^{(7)}$.

Ao vivenciar a IC, o idoso passa a estabelecer estratégias de enfrentamento dos sinais e sintomas desencadeados pela doença. Além disso, o próprio tratamento, que deve ser contínuo, também implica em mudanças de hábitos e adaptação às limitações decorrentes dos sintomas físicos, visando preservar a independência e autonomia do idoso para realizar suas atividades de vida diária.

Nesse contexto, emergiram questionamentos relativos às estratégias utilizadas pelos idosos para lidar com seu problema de saúde: quais são as repercussões da IC na qualidade de vida dos idosos? Quais estratégias são utilizadas por estes no enfrentamento destas repercussões? Como são percebidos pelos idosos os cuidados realizados pela equipe de enfermagem?

Tendo em vista o processo saúde-doença gerado pela IC na vida do idoso, este estudo teve como objetivos identificar as repercussões provocadas pela IC na qualidade de vida dos idosos; descrever as estratégias utilizadas por estes no enfrentamento destas repercussões e conhecer as percepções dos idosos acerca dos cuidados de enfermagem.

\section{O PERCURSO METODOLÓGICO}

Estudo descritivo, com abordagem qualitativa, realizado no Ambulatório de Cardiologia e nos setores de internação do Hospital Central da Aeronáutica. O HCA é um hospital militar que oferece atendimento nas áreas de Cirurgia Geral, Pediatria, Psiquiatria, Clinica Geral e Centro de Terapia Intensiva Adulto e Infantil, localizado no município do Rio de Janeiro-RJ, que atende exclusivamente militares da Força Aérea Brasileira e seus dependentes, cadastrados no Sistema de Saúde da Aeronáutica (SISAU).

Os sujeitos foram dez idosos, obedecidos os critérios de inclusão: idade igual ou superior a 60 anos, portador de IC, oficialmente cadastrado no SISAU, em acompanhamento no Ambulatório de Cardiologia ou internados nas enfermarias no período de coleta de dados; e concordar em participar da pesquisa através da assinatura do termo de consentimento livre e esclarecido. Foram excluídos os idosos que apresentavam incapacidade física ou mental registrada em prontuário médico que prejudicasse a expressão verbal e a coerência do discurso.

Atendendo ao preconizado na Resolução 196/96 do Conselho Nacional de Saúde, o projeto foi aprovado pelo Comitê de Ética em Pesquisa do Hospital Universitário Antônio Pedro, da Universidade Federal Fluminense (HUAP/UFF), com o $\mathrm{N}^{\circ}$ 005/09. Os sujeitos foram convidados a participar da pesquisa, cabendo-lhes o direito de desistência. Os que aceitaram participar do estudo foram identificados por nomes de flores escolhidas pelos próprios, visando preservar suas identidades.

Os dados foram coletados por meio de entrevista semiestruturada utilizando o mesmo roteiro em todas as entrevistas 
para orientar o pesquisador. O roteiro era composto de duas partes, uma referente aos dados de identificação como idade, sexo e outras; a segunda parte possuía quatro questões abertas relativas às repercussões provocadas pela IC na qualidade de vida, quais estratégias eram utilizadas para lidar com estas repercussões e como percebiam o cuidado realizado pela equipe de enfermagem.

A coleta de dados foi desenvolvida no mês de fevereiro de 2009. As entrevistas foram gravadas em fitas magnéticas, para preservar o conteúdo integral dos depoimentos. Após a coleta, as entrevistas foram transcritas e digitadas na íntegra pelas próprias pesquisadoras e depois submetidas à análise temática, com sucessivas leituras para identificação dos temas comuns que foram agrupados em categorias. A análise temática permitiu descobrir os núcleos de sentido presentes na comunicação dos participantes do estudo cuja presença ou frequência significavam dado relevante para a compreensão do objeto sob análise ${ }^{(8)}$.

\section{RESULTADOS E DISCUSSÃO}

\section{Caracterização dos sujeitos}

Evidenciou-se que, dos dez sujeitos desse estudo, seis estavam internados e quatro encontravam-se em acompanhamento ambulatorial. A idade destes idosos oscilou entre 60 a 90 anos, sendo cinco do sexo masculino e cinco do sexo feminino. $\mathrm{O}$ nível de escolaridade variou desde o ensino médio incompleto até o nível superior completo com pós-graduação strictu sensu.

Estudo realizado em Goiás cujo objetivo foi determinar o perfil sociodemográfico de uma amostra de 144 pacientes com IC identificou uma média de idade $61 \pm 15$ anos, sendo $54,2 \%$ do sexo masculino ${ }^{(9)}$, o que corrobora com a prevalência da IC nas idades mais avançadas em proporções semeIhantes entre homens e mulheres ${ }^{(9-10)}$.

Acerca do estado civil, todos os homens eram casados e, entre as mulheres, três eram casadas, uma era viúva e a outra separada. Dos entrevistados, três eram militares da reserva remunerada e sete eram dependentes de militares, ou seja, a pesquisa abrangeu tanto indivíduos civis quanto militares. A renda familiar destes idosos oscilava em torno de um salário mínimo até vinte e quatro salários mínimos. Dos dez idosos entrevistados, apenas um morava sozinho, os demais moravam com o conjugue e/ ou filhos. Todos os idosos referiram que não seguiam nenhuma dieta especial ou qualquer tipo de acompanhamento com profissional específico, apenas realizavam controle por conta própria.

Em relação ao tempo que os entrevistados sabiam do diagnóstico da doença, identificamos que a maioria era entre um e dezoito anos. Um deles não soube precisar há quanto tempo apresentava IC. O conhecimento do idoso sobre a sua própria doença torna-se importante, pois, além de ser um dos elementos que guiam as atividades e as intervenções terapêuticas e educativas a serem realizadas pelos profissionais de saúde, também pode influenciar na forma como o idoso enfrenta o seu processo saúde-doença.

Considerando que a evolução da IC está diretamente relacionada a fatores individuais, é necessário o acompanhamento adequado bem como a instauração de medidas de controle da evolução da IC para que a pessoa idosa possa viver bem mesmo na presença desta doença. É importante destacar que antes de planejar ações que proporcionem ao idoso com IC, informações e conhecimentos, é necessário e prioritário realizar uma escuta cuidadosa acerca das suas dúvidas e inquietações de forma a torná-lo sujeito do processo e capacitá-lo a se auto responsabilizar pela própria saúde ${ }^{(11)}$.

Considerando os dados produzidos neste estudo, três grandes categorias temáticas emergiram e serão elucidadas a seguir.

Repercussões da insuficiência cardíaca na vida dos idosos

A IC gera incapacidades e limitações físicas e sociais que repercutem diretamente na QV dos indivíduos com ocorrência de quadros agudos que levam a necessidade de hospitalização e recidivas se não houver adoção de medidas preventivas e seguimento de uma mudança no estilo de vida. A influência da IC no âmbito emocional do indivíduo com consequência direta no seu bem-estar é demonstrada na fala abaixo:

Assim, alterou muito emocionalmente. Eu vou ter mais cuidado agora! Eu acho que eu vivi de novo! Eu estava assim deprimida. A doença estava me deixando mais triste. $O$ maior pavor foi a falta de ar, é a pior que eu já senti em 70 anos. E eu já senti muitas dores, mas a falta de ar é a pior coisa! É uma sensação de afogamento, é o pior sintoma. Parecia que o mundo estava acabando para mim! (Acácia, 71 anos)

O depoimento acima aponta que a patologia traz repercussões não apenas no corpo físico, mas também no corpo psíquico. A depressão não faz parte do envelhecimento normal, mas é consequência de uma combinação de razões, como por exemplo, uma doença, sendo considerado o distúrbio psiquiátrico mais comum ${ }^{(12)}$. O bem-estar do idoso também é alterado tanto pelo impacto de ter adquirido uma doença crônica e, portanto incurável, como pelos sintomas que a mesma ocasiona. As condições crônicas e as incapacidades consequentes podem afetar significativamente o bem-estar dos idosos ${ }^{(13)}$.

Os sintomas desencadeados pela IC geram desconforto físico que impõe restrições aos hábitos rotineiros que proporcionavam prazer aos seus portadores, limitando suas atividades e repercutindo diretamente na sua autonomia e independên$\mathrm{cia}^{(14)}$, gerando alterações físicas e sociais.

Minha vida alterou, pois não posso fazer mais nada do que eu fazia. Sinto vontade de correr, mas não posso, fico cansada! Quero brincar com meus netos e não posso. (Camélia, 65 anos)

Alterou muito, porque eu não posso fazer nada porque me canso muito. Eu já trabalhei muito costurando para fábrica, mas agora com a doença não posso fazer mais nada! (Violeta, 77 anos) 
A fadiga ocorre em diversas condições agudas e crônicas, porém a frequência e intensidade de fadiga nas pessoas com IC é alta quando comparadas a não portadores dessa condição ${ }^{(15)}$. Camélia em sua fala relata que além da sintomatologia clínica, a doença também ocasionou impacto nas relações com seus familiares e com a sua rede de suporte social. A rede de suporte social é de fundamental importância no enfrentamento de uma doença crônica e proporciona ao idoso o aumento de sua autoestima, de inserção familiar e domínio sobre o seu próprio ambiente. Já o depoimento de Violeta traz outra questão importante além da sintomatologia clínica, o fato de não poder mais trabalhar devido à doença.

Os motivos que levam o aposentado a continuar ou não a trabalhar são muito complexos. Além da sensação de sentir-se útil para a família e para a sociedade, o idoso aposentado necessita, muitas vezes, permanecer no trabalho por necessidade financeira, considerando-se que, para grande maioria dos brasileiros, os valores recebidos como aposentadoria não cobrem as suas necessidades de manutenção e de seus dependentes, principalmente quando cabe ao idoso o papel de mantenedor do grupo familiar(16:5).

O avanço da IC e das manifestações clinicas da doença causam repercussões na capacidade funcional dos idosos.

Limitação funcional, principalmente quando vou amarrar o sapato, aí não dá! A tosse incomoda. Outra coisa muito difícil é ter muito remédio! Principalmente, porque a doença não regride, no máximo é estabilizada e isso apavora a gente, e aí quanto mais se sabe é pior! (Begônia, 60 anos)

Com o envelhecimento pode haver prejuízo na funcionalidade do idoso que é compreendida como a capacidade de realizar atividades de vida diária, relativas ao autocuidado, e as atividades instrumentais de vida diária, como gerenciar a casa, com autonomia e independência. O processo de saúde-doença vivenciado pelo idoso prejudica ainda mais sua capacidade funcional, tornando-o mais suscetível à dependência.

Alguns idosos quando indagados sobre possíveis repercussões geradas pela IC responderam que não houve alterações significativas.

Não alterou, porque eu não sinto essa doença. No coração eu sinto palpitação, aquela agitação, uma vez ou outra, falta de ar, mas sentir (pensando) não comer e não dormir eu não sinto. Eu não sei por que eu não entendo, eu só sei que eu a tenho. (Cravo, 80 anos)

Algumas vezes o idoso não percebe as repercussões da IC na sua qualidade de vida porque as pessoas possuem mecanismos e capacidades diferentes para o enfrentamento de uma doença crônica que se manifesta de acordo com a visão de mundo de cada pessoa, visto que cada indivíduo possui uma história de vida única, dotada de crenças e valores próprios ${ }^{(17)}$. A negação de influências da IC na vida dos idosos do estudo pode estar diretamente associada ao déficit de conhecimento acerca da patologia, dos seus sinais e sintomas.

O tratamento da IC envolve mudanças nos hábitos e no estilo de vida, o uso contínuo de medicamentos para controle dos sintomas. O aumento no consumo de medicamentos promove um aumento nos gastos com orçamento doméstico o que leva a alterações na vida dos idosos.

A minha vida alterou muito porque adquiri dívidas, entendeu? Eu tinha uma firma de imóveis e fechei esse imóvel porque fiquei doente e sem dinheiro. A doença gera gastos com remédios. Eu fiquei internado e aí perdi tudo! (Dália, 74 anos)

Muitas vezes o idoso ao adoecer torna-se menos produtivo economicamente, o que reduz seu poder aquisitivo e prejudica o estabelecimento e/ou manutenção do tratamento, dificultando sua recuperação, levando a complicações e constantes internações hospitalares. Torna-se importante propiciar para este idoso, oportunidades para que ele aprenda a lidar com as mudanças que ocorrem no seu corpo, tirando proveito da sua condição, conquistando sua autonomia, sentindo-se sujeito da sua própria história.

\section{Estratégias utilizadas frente às repercussões da Insuficiên- cia Cardíaca}

A forma como cada indivíduo percebe a doença é singular, sendo dependente da sua história de vida. A vida é mesclada de situações favoráveis ou não, e a forma como reagirmos pode ser determinante na produção de doenças crônicas.

Há duas formas de enfrentamento: o enfrentamento focalizado no problema, que ocorre quando a situação estressante é vista como reversível, logo pode ser removida e/ou alterada e o enfrentamento paliativo que é aquele focalizado na emoção cujo estressor é visto como inalterável. Essas duas formas de enfrentamento são inter-relacionadas em uma relação recíproca e dinâmica entre emoção e enfrentamento. É importante mencionar que o conhecimento acerca da doença facilita a adesão do cliente ao tratamento e permite ao mesmo criar estratégias para conviver melhor com ela, devendo ser uma das estratégias a ser implementada no grupo de pessoas em condições crônicas de saúde ${ }^{(14)}$.

Alguns idosos estabeleceram, como estratégias de enfrentamento da IC, a prática de atividades e cuidados considerados fontes de prazer na convivência diária com a doença em suas vidas.

[...] fazer alguma coisa que eu adoro, como ir ao supermercado, ir à praia, realizar compras, porque estas coisas me estimulam muito. Ir à igreja me faz muito bem, aquela visita a Jesus! Tudo isso me dá sentido. Quando eu tenho falta de ar eu faço nebulização e fico sentada no sofá, esquento um leitinho no microondas com pouquinho de açúcar tomo e melhoro! (Acácia, 71 anos)

A realização de atividades que proporcionem prazer contribui para amenizar os eventos críticos da vida e representam 
um enfretamento baseado na regulação afetiva que engloba a otimização afetiva e a complexidade afetiva $^{(13)}$. O enfrentamento focalizado na emoção se caracteriza pelas estratégias de sentimento de fé, pelo trabalho, pela busca da família ou de outras pessoas significantes. A fé, que é um sentimento de confiança no que se deseja, está arraigada em nossa cultura e é tão necessária quanto são os outros modos de enfrentamento sendo imprescindível conhecer as crenças e os costumes dos clientes ao se planejar o cuidado de enfermagem ${ }^{(14)}$. A manutenção do convívio social também representa uma estratégia positiva que auxilia o idoso na convivência com a IC.

Não alterou nada, eu continuo mantendo os ciclos de relação de amizade, eu encontro com minhas amigas toda semana. Eu tenho vários grupos de amigas, um uma vez por mês, outro uma vez por semana. Eu sou uma pessoa que não gosto ficar sozinha. As minhas amizades me fazem um bem enorme. Eu não sei viver só. Você tem que se adaptar aquilo que tem. Você também não pode chegar aos 90 anos, como você era aos 50, 60 anos. Mesmo com a deficiência, você tem que acreditar que são as peculiaridades da idade. (Margarida, 90 anos)

A rede de suporte social representada pelas amizades permite ao idoso enfrentar melhor a doença, aceitando-a, mas sem sentir-se inativo ou prejudicado porque possui uma doença crônica. Há alguns idosos que passam a praticar atividades que Ihes mantém ativos como forma paliativa de enfrentar o problema.

Eu procuro andar sempre que possível, jogar xadrez no computador, manter-se ativo. (Begônia, 60 anos)

O enfrentamento paliativo pode ser interpretado pela teoria da continuidade que relata como os idosos mantêm as suas estruturas internas e externas preexistentes. A continuidade interna inclui memória, ideias, afeto, habilidade e experiências e as externas incluem a manutenção do domínio, competência, auto integridade e autoestima ${ }^{(14)}$. Alguns idosos afirmaram não adotar nenhuma estratégia.

Eu não tenho! Deixei de trabalhar devido à idade. Parei de trabalhar devido à natureza, eu sentia falta de ar quando eu subia na escada. (Cravo, 80 anos)

A manutenção de uma boa qualidade de vida e bem-estar por parte de idosos que vivem em seu cotidiano com uma doença crônica que acarreta uma série de dificuldades e diversidades representa um paradoxo. Isso é possível porque o enfrentamento produzido depende de um balanço entre o corpo e mente e da manutenção de relações harmoniosas nos contextos sociais e pessoais ${ }^{(11)}$. Evidencia-se que a forma como o indivíduo sente, percebe e reage aos problemas decorrentes da doença bem como desenvolve mecanismos de enfrentamento vai depender do seu contexto social, econômico, cultural e dos seus recursos psicológicos que foram adquiridos ao longo da vida, fruto das suas relações com o meio.
Os profissionais de saúde e dentre estes os enfermeiros, durante o cuidado aos idosos que convivem com a IC, devem estar atentos às formas de enfrentamento adotadas por estes clientes, para que possam estimular recursos positivos que contribuam para uma vida com qualidade.

\section{O cuidado de enfermagem ao idoso com insuficiência cardíaca}

O cuidar em enfermagem busca ajudar o cliente a identificar suas necessidades frente aos problemas reais e potenciais decorrentes da IC, implementando ações educativas que promovam melhoria na sua QV. É o compromisso com o cuidado existencial, a autovalorização, a cidadania do outro e da própria pessoa que cuida. $\mathrm{O}$ cuidado existencial ocorre quando aquele que cuida compreende o mundo subjetivo do paciente, o que permite respeitar a liberdade de ser de cada um ${ }^{(18)}$. É nesta perspectiva que a enfermagem gerontológica deve atuar, valorizando todas as dimensões que envolvem o idoso.

Entre as ações de enfermagem identificadas pelos sujeitos desta pesquisa, predominaram aquelas relacionadas ao nível afetivo, como a atenção e o carinho na forma como eles se sentem cuidados.

Pessoal da enfermagem é o máximo comigo, agradeço muito! A atenção que eles têm comigo é o máximo! (Dália, 74 anos)

A enfermagem é ótima, o carinho que eles têm com a gente, é pouca gente trabalhando, mas mesmo assim eles dão maior atenção. (Lírio, 65 anos)

O cuidado na perspectiva da enfermagem significa atender as necessidades do cliente com sensibilidade, presteza e solidariedade, mediante ações e atitudes que promovam o conforto e o bem-estar. O cuidado de enfermagem utilizando-se da forma verbal e não verbal objetiva transmitir tranquilidade, carinho, conforto, bem-estar, atenção e segurança ao paciente ${ }^{(18)}$.

São maravilhosos! Fazer mais do que fazem?! Eu acho que é impossível. Eles dão assistência, eles estão aqui. É medicamento, uma palavra de apoio, vêem que o paciente está necessitando de alguma ajuda e dão uma palavra na hora certa. Eu tenho muito a agradecer a enfermagem. Vou falar mais o quê? Eles são maravilhosos! (Camélia, 65 anos)

O cuidado ao paciente envolve uma boa comunicação e o estabelecimento de uma relação interpessoal saudável, que proporcione confiança e apoio o que permite que as necessidades do cliente, famílias e comunidades sejam identificadas e atendidas. O plano de alta hospitalar também é um cuidado oferecido pelo enfermeiro que foi destacado como primordial no cuidado ao idoso com IC.

[...] dar todas as indicações de como eu vou viver a partir de agora, um plano de orientação. É lógico que agora não farei mais as coisas que eu fazia quando eu era jovem, principalmente a alimentação, pois eu fui habituado a comer de 
TU-DO. Nunca restringi alimentação, se não fosse a doença, eu ia continuar comendo de tudo. (Rosa, 74 anos)

A orientação planejada da enfermeira na alta hospitalar é uma fase no processo de cuidar em enfermagem constituindo assim uma forma organizada de expressar os cuidados de enfermagem relativos à alimentação, repouso, atividade física, medicamentos, cuidados especiais relativos à patologia e as condições particulares do paciente.

A enfermeira, ao cuidar dos idosos em condições crônicas, deve reconhecer suas especificidades para saber identificar e compreender suas necessidades, conhecimentos e experiências, propondo e criando espaços em que os idosos troquem suas experiências, conhecimentos e pratiquem a sociabilidade e a espiritualidade, compartilhando situações adversas e favoráveis da vida adquirindo desta forma maneiras saudáveis de enfrentamento dos seus problemas de saúde ${ }^{(14)}$.

Essas orientações dadas pela enfermeira no momento da alta hospitalar podem elucidar muitas indagações acerca do processo saúde-doença o que ajudará o idoso a viver melhor com a doença contribuindo para a melhoria na QV e prevenção de possíveis complicações que podem levar a reinternações.

Assim, cuidar do idoso com IC implica em conhecer sua experiência com a doença, avaliando suas repercussões na capacidade física e autonomia do mesmo para as atividades de vida diária, estimulando suas potencialidades e oferecendo suporte para que ele apreenda na experiência cotidiana formas de se cuidar. Esse cuidado exige habilidades e competências do enfermeiro para identificar as especificidades do idoso com IC, em uma perspectiva de cuidado integral que contribua para a QV destes clientes.

\section{CONSIDERAÇÕES FINAIS}

Os dados desta pesquisa apontaram que além da influência no âmbito emocional do idoso, a IC também promove alterações no âmbito social e familiar, pois as relações dele com seus familiares e sua rede de suporte social também ficam alteradas. A capacidade funcional também fica prejudicada na presença da IC, o que torna o indivíduo mais suscetível à dependência e a perda da autonomia. Também foram identificadas repercussões no campo financeiro, devido à cronicidade da doença, aumento dos custos com remédios, consultas e exames o que pode prejudicar a adesão ao tratamento.

Em relação às estratégias de enfrentamento utilizadas pelos idosos do estudo, o enfrentamento paliativo aparece como um dos principais. Alguns idosos também citaram o enfrentamento focalizado na emoção, e outros afirmaram não adotar nenhuma estratégia de enfrentamento, o que demonstra que a forma como o indivíduo desenvolve estes mecanismos vai depender do seu contexto social, econômico, cultural e dos recursos psicológicos que foram adquiridos ao longo da vida, fruto das suas relações com o meio.

Como ações de enfermagem essenciais no cuidado têm-se a atenção, o carinho e o apoio da enfermeira e da equipe de enfermagem ao idoso e sua família que representam fontes de apoio emocional e bem-estar favorecendo a recuperação desses clientes.

A limitação deste estudo versa sobre a abordagem da pesquisa que embora possa ter sido limitadora no aspecto da quantificação do impacto da IC na QV dos idosos, mostrou-se sensível à individualidade e complexidade da mesma, através do uso da entrevista como técnica de coleta de dados. Outra limitação foi o número dos sujeitos do estudo que deve ser ampliado de forma a captar mais profundamente as influências da IC e as estratégias de enfrentamento que os idosos utilizam para conviver com essa doença no cotidiano.

No que tange às contribuições, espera-se que os resultados desta pesquisa possam contribuir para novos investimentos profissionais e institucionais que favoreçam a recuperação, manutenção e promoção da saúde de clientes idosos com IC que necessitam ser respeitados em sua singularidade e complexidade em todos os âmbitos da nossa sociedade, além de possibilitar novos caminhos para o ensino e pesquisa, sobretudo no cenário militar.

\section{REFERÊNCIAS}

1. Soares DAS, Toledo JAS, Santos LF, Lima RMB, Galdeano LE. Qualidade de vida de portadores de insuficiência cardíaca. Acta Paul. Enferm. 2008;21(2):243-8.

2. Hurst JW. Exame inicial para o diagnóstico cardiovascular. Rio de Janeiro: Revinter; 2000.

3. IBGE. Indicadores Sociodemográficos e de Saúde no Brasil 2010. [online] Pesquisa Nacional por Amostra de Domicílios (PNAD) - 2009 [citado em 06 Out 2010]. Disponível em: http://www.ibge.gov.br/home/presidencia/noticias/noticia _visualiza.php?id_noticia $=1708$ \&id_pagina $=1$

4. Brunini TMC, Ribeiro ACM. Mecanismos fisiopatológicos na insuficiência cardíaca. In: Ferreira ME. Insuficiência cardíaca. Rio de Janeiro: Rubio; 2007. p. 1-2.

5. Nogueira IDB, Servantes DM, Nogueira PAMS, Pelcerman
A, Salvetti XM, Salles F et al. Correlação entre qualidade de vida e capacidade funcional na insuficiência cardíaca. Arq. Bras. Cardiol. 2010;95(2):238-243.

6. Scattolin FAA, Diogo MJD, Colombo RCR. Correlação entre instrumentos de qualidade de vida relacionada à saúde e independência funcional em idosos com insuficiência cardíaca. Cad. Saúde Pública 2008;23(11):2705-15.

7. Mantovani MF, Ulbrich EM, Pinnoti S, Giacomozzi LM, Labronici LM, Sarquis LMM. O significado e a representação da doença crônica: conhecimento do portador de hipertensão arterial acerca de sua enfermidade. Cogitare enferm. 2008;13(3):336-42.

8. Minayo MCS. O desafio do conhecimento: pesquisa qualitativa. 12a ed. São Paulo: Hucitec - Abrasco; 2010. 
9. Nogueira PR, Rassi S, Corrêa KS. Perfil epidemiológico, clínico e terapêutico da insuficiência cardíaca em hospital terciário. Arq. Bras. Cardiol. 2010;95(3):392-398.

10. Ho KKL, Pinsky JL, Kannel WB, Levy D. The epidemiology of heart failure: the Framingham study. J Am Coll Cardiol. 1993;22(Suppl A):6-13.

11. Oliveira, DLLC. A enfermagem e suas apostas no autocuidado: investimentos emancipatórios ou práticas de sujeição? Rev. Bras. Enferm. 2011;64(1):185-8.

12. Roach S. O aparelho cardiovascular. In: Roach S. Introdução à enfermagem gerontológica. Rio de Janeiro: Guanabara Koogan; 2003. p. 191-210.

13. Rabelo DF, Neri AL. Recursos Psicológicos e ajustamento pessoal frente à incapacidade funcional na velhice. Psicol. Estud. 2005;10(3):403-12.

14. Trentini M, Silva SH, Valle ML, Hammerschmidt KSA. Enfrentamento de situações adversas e favoráveis por pessoas idosas em condições crônicas de saúde. Rev. Latino-Am. Enfermagem 2005;13 (1):38-45.

15. Fini A, Cruz DALM. Propriedades psicométricas da Dutch Fatigue Scale e Dutch Exertion Fatigue Scale: versão brasileira. Rev. Bras. Enferm. 2010;63(2):216-221.

16. Bulla LC, kaefer, CO. Trabalho e aposentadoria: as repercussões sociais na vida do idoso aposentado. Revista Virtual Textos \& Contextos [periódico online]2003[citado em 20 Out 2011];2(Ano II):1-8. Disponível em: http:// revistaseletronicas.pucrs.br/ojs/index.php/fass/article/ viewFile/957/737

17. Santos ACS. O Idoso com insuficiência cardíaca: em busca da qualidade de vida [monografia]. Niterói (RJ): Escola de Enfermagem Aurora de Afonso Costa, Universidade Federal Fluminense; 2006.

18. Caldas CP. A dimensão existencial da pessoa idosa e seu cuidador. Textos envelhecimento 2000;3(4):29-41. 